\title{
Safety of sildenafil in premature infants with severe bronchopulmonary dysplasia (SILDI-SAFE): a multicenter, randomized, placebo-controlled, sequential dose- escalating, double-masked, safety study
}

\author{
Simone Schneider ${ }^{1}$, Mary Bailey², Tracy Spears², Charles R. Esther Jr ${ }^{1}$, Matthew M. Laughon',
} Christoph P. Hornik ${ }^{2,3}$ and Wesley Jackson ${ }^{1 *}$

\begin{abstract}
Background: Pulmonary hypertension is a deadly complication of bronchopulmonary dysplasia, the most common pulmonary morbidity of prematurity. Despite these catastrophic consequences, no evidence-based therapies are available for the prevention of pulmonary hypertension in this population. Sildenafil is a potent pulmonary vasodilator approved by the US Food and Drug Administration for the treatment of pulmonary hypertension in adults. Preclinical models suggest a beneficial effect of sildenafil on premature lungs through improved alveolarization and preserved vascular development. Sildenafil may therefore prevent the development of pulmonary hypertension associated with lung disease of prematurity by reducing pulmonary vascular remodeling and lowering pulmonary vascular resistance; however, clinical trial evidence is needed. The present study, supported by the National Institutes of Health's National Heart Lung and Blood Institute, will generate safety, pharmacokinetics, and preliminary effectiveness data on sildenafil in a population of premature infants with severe bronchopulmonary dysplasia at risk for pulmonary hypertension.

Methods: We have designed a multicenter, randomized, placebo-controlled, sequential dose-escalating, doublemasked, safety trial of sildenafil in premature infants with severe bronchopulmonary dysplasia. We will randomize 120 premature infants $<29$ weeks gestational age with severe bronchopulmonary dysplasia at 32-40 weeks postmenstrual age in a dose-escalating approach 3:1 (sildenafil: placebo) sequentially into each of 3 cohorts at $\sim 30$ clinical sites. Participants will receive up to 34 days of study drug, followed by 28 days of safety monitoring. The primary outcome will be safety as determined by incidence of hypotension. Secondary outcomes will include pharmacokinetics and preliminary effectiveness of sildenafil based on presence or absence of pulmonary hypertension diagnosed by echocardiography at the end of treatment period.

(Continued on next page)
\end{abstract}

\footnotetext{
* Correspondence: wesley.jackson@unc.edu

'Department of Pediatrics, School of Medicine, The University of North Carolina at Chapel Hill, Chapel Hill, NC, USA

Full list of author information is available at the end of the article
}

(c) The Author(s). 2020 Open Access This article is licensed under a Creative Commons Attribution 4.0 International License, which permits use, sharing, adaptation, distribution and reproduction in any medium or format, as long as you give appropriate credit to the original author(s) and the source, provide a link to the Creative Commons licence, and indicate if changes were made. The images or other third party material in this article are included in the article's Creative Commons licence, unless indicated otherwise in a credit line to the material. If material is not included in the article's Creative Commons licence and your intended use is not permitted by statutory regulation or exceeds the permitted use, you will need to obtain permission directly from the copyright holder. To view a copy of this licence, visit http://creativecommons.org/licenses/by/4.0/ The Creative Commons Public Domain Dedication waiver (http://creativecommons.org/publicdomain/zero/1.0/) applies to the data made available in this article, unless otherwise stated in a credit line to the data. 
(Continued from previous page)

Discussion: Sildenafil is a promising intervention to prevent the development of pulmonary hypertension in premature infants with bronchopulmonary dysplasia. Clinical trials of sildenafil specifically designed for premature infants are urgently needed. The current study will make substantial contributions to scientific knowledge of the safety of sildenafil in premature infants at risk for pulmonary hypertension. Results from the study will be used by investigators to inform the design of a pivotal efficacy trial.

Trial registration: ClinicalTrials.gov NCT04447989. Registered 25 June 2020.

Keywords: Pulmonary hypertension, Premature infant, Bronchopulmonary dysplasia, Randomized control trial, Sildenafil, Hypotension, Mean arterial pressure, Echocardiogram

\section{Background}

Each year in the United States, over 50,000 infants are born < 29 weeks gestational age (GA), of whom nearly $40 \%$ develop bronchopulmonary dysplasia (BPD) [1]. The severity of BPD is defined by the level of respiratory support at 36 weeks postmenstrual age (PMA) [2]. Premature infants with severe BPD are particularly challenging to manage and often suffer from multiple complications and comorbidities, including prolonged hospitalization, need for home respiratory support, and death [3-5]. A common comorbidity in infants with severe $B P D$ is pulmonary hypertension $(\mathrm{PH})$. Infants with BPD-associated $\mathrm{PH}$ have significantly higher mortality compared to infants with BPD who do not develop $\mathrm{PH}$ [3-5].

The temporal relationship between $\mathrm{BPD}$ and $\mathrm{PH}$ in premature infants is complex. Approximately 20\% of infants with severe BPD develop $\mathrm{PH}$, and nearly half of these infants die, most often due to right-sided heart failure [4, 6-9]. Early PH in the first post-natal week is a risk factor for increased BPD severity [6]. However, most infants at risk for BPD develop PH later in life: one prospective study of extremely low birth weight infants found that $65 \%$ of infants with $\mathrm{BPD}$-related $\mathrm{PH}$ had no evidence of $\mathrm{PH}$ on screening echocardiograms obtained at 4-6 weeks of life [10].

There are currently no US Food and Drug Administration (FDA) approved or evidence-based drugs to prevent or treat $\mathrm{PH}$ in premature infants with BPD. Sildenafil is a potent inhibitor of phosphodiesterase type 5, the predominant isoform in the lung, which metabolizes cyclic guanosine monophosphate and produces pulmonary vasodilation by potentiating the effects of endogenous nitric oxide. The FDA has approved the use of sildenafil for treatment of pulmonary arterial hypertension in adults to improve exercise ability and delay clinical worsening [11]. The FDA does not recommend sildenafil for $\mathrm{PH}$ in children 1 to 17 years of age and there are no recommendations regarding its use in neonates and infants.

The objectives of this trial are to characterize the safety and pharmacokinetics (PK) of sildenafil in premature infants with severe $\mathrm{BPD}$, as well as provide data on efficacy for the prevention of $\mathrm{PH}$ in this population. Funding for this study is provided by the National Institutes of Health's National Heart Lung and Blood Institute (NHLBI; R61HL147833).

\section{Methods/design}

This is a multicenter, randomized, placebo-controlled, sequential dose-escalating, double masked, safety study of sildenafil in premature infants with severe BPD. The primary objective of the study is to describe the safety of sildenafil as determined by incidence of hypotension. We will analyze the PK and preliminary effectiveness of sildenafil in the prevention of $\mathrm{PH}$ in premature infants with severe BPD as secondary objectives.

\section{Study subjects}

Premature infants in the Neonatal Intensive Care Unit (NICU) will be randomized into 3 cohorts with a ratio of 3:1 (sildenafil:placebo). Each sequential cohort will receive a higher dose of sildenafil for those randomized to the treatment arm (Table 1). There will be approximately 40 randomized and dosed participants in each cohort for a total of up to 120 participants.

To be eligible to participate in this study, an infant must meet all of the following inclusion and exclusion criteria:

Table 1 Number of Participants (N) and Dosing Scheme

\begin{tabular}{|c|c|c|c|c|c|}
\hline \multirow[t]{2}{*}{ Cohort $^{a}$} & \multicolumn{2}{|c|}{ Treatment Group } & \multicolumn{2}{|c|}{$\begin{array}{l}\text { Sildenafil Dosing } \\
\text { (mg/kg q } 8 \mathrm{~h})\end{array}$} & \multirow[t]{2}{*}{$\begin{array}{l}\text { Total } \\
\text { (N) }\end{array}$} \\
\hline & Placebo (N) & Sildenafil (N) & IV & Enteral & \\
\hline 1 & 10 & 30 & 0.5 & 1 & 40 \\
\hline 2 & 10 & 30 & 1 & 2 & 40 \\
\hline 3 & 10 & 30 & 2 & 4 & 40 \\
\hline
\end{tabular}

Abbreviations: $I V$ intravenous, $N$ number of participants, $q$ every

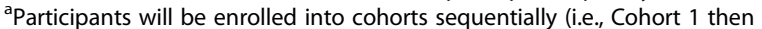
Cohort 2 then Cohort 3) based on safety

${ }^{\mathrm{b}}$ Route of administration should be via IV route if patient has an IV and IV administration is feasible. However, route of administration, IV or enteral, is left to investigator discretion 


\section{Inclusion criteria}

1 Documented informed consent from parent or guardian, prior to study procedures

$2<29$ weeks GA at birth

3 32-40 weeks PMA

4 Receiving respiratory support at enrollment:

- If 32-36 weeks PMA: mechanical ventilation (high frequency or conventional)

- If > 36-40 weeks PMA: mechanical ventilation (high frequency or conventional) OR continuous positive airway pressure (CPAP)

Note:

- Criteria 3 and 4 define severe BPD for the purposes of this study.

- CPAP is defined as any of the following:

- Nasal cannula > 2 liters per minute (LPM)

- Nasal continuous positive airway pressure (NCPA P)

- Nasal intermittent positive pressure ventilation (NIPPV)

- Noninvasive neurally adjusted ventilatory assist (NAVA)

- Any other device designed to provide positive pressure through a nasal device (e.g., RAM cannula, etc.)

\section{Exclusion criteria}

1 Previous enrollment and dosing in this study

2 Previous exposure to sildenafil within 7 days prior to randomization

3 Previous exposure to vasopressors within $24 \mathrm{~h}$ prior to randomization

4 Previous exposure to inhaled nitric oxide within 24 $\mathrm{h}$ prior to randomization

5 Previous exposure to milrinone within $24 \mathrm{~h}$ prior to randomization

6 Evidence of $\mathrm{PH}$ or moderate/large patent ductus arteriosus (PDA) on the most recent echocardiogram performed within 14 days prior to randomization

7 Known major congenital heart defect requiring medical or surgical intervention in the neonatal period

8 Known allergy to sildenafil

9 Known sickle cell disease

10 Aspartate aminotransferase (AST) $>225 \mathrm{U} / \mathrm{L}<72 \mathrm{~h}$ prior to randomization

11 Alanine aminotransferase (ALT) $>150 \mathrm{U} / \mathrm{L}<72 \mathrm{~h}$ prior to randomization
12 Any condition that would make the participant, in the opinion of the investigator, unsuitable for the study

\section{Recruitment sites}

Approximately 30 sites will be recruiting patients for the study. Sites were selected based on prior participation in infant trials including those conducted by the National Institute of Child Health and Human Development Pediatric Trials Network. These research teams have extensive experience interacting with and enrolling the target population. Prior to and during the enrollment period for the study, sites will participate in conference calls to discuss recruitment and retention plans, including strategies for building rapport, increasing awareness, and enrolling the study population.

Potential subjects will be identified in the NICU at participating sites. Due to the nature of the condition, a minority population is intrinsically overrepresented (minority population represents $30-40 \%$ of infants with BPD; https://www.marchofdimes.org/peristats). Therefore, the study team does not anticipate difficulty with inclusion of individuals commonly underrepresented in research studies.

\section{Baseline screening}

Prior to initiation of the first dose of study drug, participant and maternal demographics, mean arterial pressures (MAP), concomitant medications, laboratory evaluations (AST, ALT; when available, values for platelets, phosphorus, chloride, alkaline phosphatase, and caffeine level), and respiratory assessments will be collected (Additional file 1: Appendix Table 1). Echocardiogram reports will be collected if performed per local standard of care < 14 days prior to start of study drug. If not performed per local standard of care $<14$ days prior to start of study drug, an echocardiogram will be required to confirm eligibility.

\section{Treatment protocol Randomization}

Participants will be centrally randomized via electronic data capture to maintain a 3:1 ratio within each cohort of sildenafil $(n=30)$ to placebo $(n=10)$. Participants from a multiple gestation will be randomized independently as they become eligible. Study randomization codes and replacement codes for all cohorts will be generated and uploaded prior to the enrollment of the first participant. If more than one infant from a multiple gestation is randomized, the sibling relationship between participants will be collected. Investigators are encouraged to use IV dosing if the patient has IV access. If the participant does not have an IV or if the clinical team elects to use enteral dosing, then the dose of study drug will be 
twice that of the IV dosing (from the product label). Placebo will be administered either enterally or IV in the same manner. Infants randomized to the placebo treatment group will receive the equivalent volume of dextrose $5 \%$.

\section{Masking procedures}

Study drug and placebo will be acquired from the hospital pharmacy using commercially available preparations. The investigational pharmacist will be unmasked, and will prepare drug-appropriate sized syringes in a masked manner (e.g., amber syringe). Staff accessing participant outcomes will be masked to treatment. Drug or placebo will be administered by the bedside nurse. Intravenous doses will be administered as an infusion over $60 \mathrm{~min}$ followed by $30 \mathrm{~min}$ of flush. Enteral doses will be administered with feedings.

\section{Dose escalation}

Dose escalation of study drug will occur if the infant continues to receive exogenous oxygen or respiratory support (nasal cannula or positive pressure from any device) and AST $<225 \mathrm{U} / \mathrm{L}$ and ALT $<150 \mathrm{U} / \mathrm{L}$ within the 7 days prior to the last dose increase. Patients meeting these criteria will escalate their dose until goal dose is reached, and then receive 28 days of drug therapy at goal dosing prior to weaning or discontinuation of the study drug. (Table 2).

\section{Treatment period monitoring}

The treatment period will include Days 1-28 or last day of study drug if early withdrawal of study drug. During the treatment period, the following information will be collected and recorded while the participant is on study drug: all MAP values obtained $24 \mathrm{~h}$ after the first dose of study drug and serial MAP values around the time of study drug administration, concomitant medications, daily respiratory assessments, laboratory evaluations (AST, ALT; when available, values for platelets, phosphorus, chloride, alkaline phosphatase, and caffeine level), PK sampling, echocardiograms and cardiac catheterization reports, if performed per local standard of care, and serious adverse events (SAEs), including events of special interest.

\section{Weaning}

Sildenafil is commonly used at higher doses to treat $\mathrm{PH}$ in infants. In clinical practice, it is recommended to wean from these higher doses to prevent rebound effects that may be seen with abrupt discontinuation of sildenafil. Although sildenafil used in this study is for prevention of $\mathrm{PH}$, the target doses in Cohorts 2 and 3 are similar to those used for the treatment of $\mathrm{PH}$.

For Cohorts 2 and 3, weaning of study sildenafil or placebo will begin following the last study dose on Day 28 or if the dose escalates to a dose of $\geq 0.5 \mathrm{mg} / \mathrm{kg}$ IV or $\geq 1 \mathrm{mg} / \mathrm{kg}$ enteral and the participant is withdrawn from the study. Study drug will be weaned by $25 \%$ of the last dose every 2 days until off. Participants should complete weaning after 6 days (Table 3). Weaning will not be performed for participants in Cohort 1 as the risk of rebound effects is likely minimal for infants receiving low dose sildenafil.

\section{Follow-up period}

The follow-up period will include Days 1-28 after the last study drug dose; last study drug dose may occur prior to Day 28 for those participants who withdraw from study drug early; on Day 28 for those participants who complete the full treatment period; or after last weaning dose for those participants who require weaning. The following information will be reported during the follow-up period: the lowest valid MAP value on follow-up days $1,14,21$, and 28 , daily respiratory

Table 2 Dose Escalations for Each Sequential Cohort

\begin{tabular}{|c|c|c|c|c|c|c|c|c|}
\hline & \multicolumn{8}{|c|}{ Study Days (Dose Numbers) } \\
\hline & $1-2(1-6)$ & $3-4(7-12)$ & $5-6(13-18)$ & $7-8(19-24)$ & $9-10(25-30)$ & $11-12(31-36)$ & $13-14(37-42)$ & $15+(43+)$ \\
\hline \multicolumn{9}{|c|}{ Sildenafil Dose (mg/kg) } \\
\hline \multicolumn{9}{|c|}{ Cohort 1} \\
\hline IV & 0.25 & 0.5 & 0.5 & 0.5 & 0.5 & 0.5 & 0.5 & 0.5 \\
\hline Enteral & 0.5 & 1 & 1 & 1 & 1 & 1 & 1 & 1 \\
\hline \multicolumn{9}{|c|}{ Cohort 2} \\
\hline IV & 0.25 & 0.5 & 0.75 & 1 & 1 & 1 & 1 & 1 \\
\hline Enteral & 0.5 & 1 & 1.5 & 2 & 2 & 2 & 2 & 2 \\
\hline \multicolumn{9}{|c|}{ Cohort 3} \\
\hline IV & 0.25 & 0.5 & 0.75 & 1 & 1.5 & 1.75 & 2 & 2 \\
\hline Enteral & 0.5 & 1 & 1.5 & 2 & 3 & 3.5 & 4 & 4 \\
\hline
\end{tabular}


Table 3 Weaning Schedule for Study Sildenafil or Placebo in Cohorts 2 and 3

\begin{tabular}{lll}
\hline Weaning Days & IV & Enteral \\
\hline $1-2$ & $75 \%$ of last study dose & $75 \%$ of last study dose \\
$3-4$ & $50 \%$ of last study dose & $50 \%$ of last study dose \\
$5-6$ & $25 \%$ of last study dose & $25 \%$ of last study dose \\
7 & Discontinue & Discontinue \\
\hline
\end{tabular}

Abbreviation: $I V$ intravenous

assessments, laboratory evaluations, concomitant medications of special interest, follow-up echocardiograms, and SAEs, including events of special interest.

\section{Respiratory assessments}

Respiratory assessments will be recorded daily during treatment, weaning, and follow-up periods. The following information will be recorded from the medical chart: maximum daily fraction of inspired of oxygen (FiO2), highest level of ventilation type (i.e., high-frequency ventilation, conventional ventilation, non-invasive support, no support), and highest mean airway pressure, if receiving invasive mechanical ventilation.

\section{Data management}

All clinical report forms and laboratory reports must be reviewed by the clinical team and data entry staff, who will ensure that they are accurate and complete. The DCRI will be responsible for data management, quality review, analysis, and reporting of study data. Clinical data will be entered into an internet data entry system provided by the DCC. The data system includes password protection and internal quality checks, such as automatic range checks, to identify data that appear inconsistent, incomplete, or inaccurate.

\section{Safety/adverse event policy}

The Duke Clinical Research Institute (DCRI) Safety Surveillance team will be overseeing real-time significant adverse event collection, evaluation, and expedited regulatory reporting for this study. Site investigators will record all SAEs and ESIs from the time of the first studyspecific procedure through 28 days after the last dose of the study drug. A clinician not involved in the study protocol will be appointed as a medical monitor and will review all valid SAEs and hypotension events at the time they are reported. If safety concerns are identified, the medical monitor may request a meeting of the Data and Safety Monitoring Board (DSMB) to review safety data. All hypotension and SAEs (study-drug related or not) will be followed until resolution. Regular meetings will be held throughout the study to review all safety events. An unscheduled DSMB review of safety data will be triggered if more than 3 patients in a cohort have their treatment discontinued or an infusion stopped due to the same type of event or 3 patients in a cohort have a suspected adverse reaction. Enrollment in the study will be suspended during this review. If the sponsor confirms that an SAE is related and unexpected, a MedWatch will be submitted to the FDA and the treatment assignment requires unblinding. The site investigator will provide direct access to all trial-related locations, source data/ documents, clinical report forms, and reports for the purpose of monitoring and auditing by the sponsor, and inspection by local and regulatory authorities.

\section{Primary outcome measures}

Safety of sildenafil will be determined by the incidence of hypotension experienced by the participants through 28 days post last dose of study drug. Hypotension will be defined as any clinically significant low blood pressure event deemed by the treating physician to require intervention with a fluid bolus or the initiation or escalation of inotropic, vasopressor, or systemic steroid therapy with the specific intent to raise blood pressure.

MAP values will be obtained at a minimum of $2 \mathrm{~h}, 1 \mathrm{~h}$, and 15 min prior to the first dose of study drug or first dose of an escalation. For IV formulations MAP will be obtained at the start of the infusion, every 15 min during the infusion, and at the end of the infusion. MAP will also be assessed at $15 \mathrm{~min}, 30 \mathrm{~min}$, hourly for $4 \mathrm{~h}$, and one additional measurement prior to the subsequent dose. For enteral doses MAP will be obtained at the start of the enteral administration, then every $15 \mathrm{~min}$ for 90 $\mathrm{min}$, then every $30 \mathrm{~min}$ for $60 \mathrm{~min}$, then hourly for $4 \mathrm{~h}$, then once in the remaining $2 \mathrm{~h}$ prior to the next dose. For subsequent IV or enteral daily doses, the lowest valid MAP value should be recorded.

\section{Secondary outcome measures Pharmacokinetics}

Blood samples will be collected after any dose following the completion of 7 days $(168 \mathrm{~h})$ of study drug administration. A population PK analysis will be performed. Using the final population PK model, empirical Bayesian estimates of clearance, volume of distribution, and halflife will be generated for each participant and used to calculate exposure metrics (e.g. area under the time concentration curve, maximum concentration).

\section{Preliminary effectiveness}

The presence or absence of $\mathrm{PH}$ will be determined using central review by blinded pediatric cardiologists of echocardiograms performed at the end of the study period. The echocardiograms will be reviewed using a standardized reading protocol for detection of $\mathrm{PH}$ [12]. The developed population PK model will be linked to the diagnosis of $\mathrm{PH}$ made by echocardiography at the end of 
the study period to develop a population PK/pharmacodynamics (PD) model. We will use the model to simulate the effect of variable sildenafil exposures on the probability of developing $\mathrm{PH}$.

\section{Tertiary outcome measures}

\section{Global rank}

A Global Rank Score (GRS) will be determined for each participant based on pre-specified endpoints (Additional file 2: Appendix Table 2). All endpoints for all participants will be ranked by severity $(1=$ worst $)$. Each participant will receive a single rank based on the lowest ranking endpoint that they experienced during their participation in the study. Wilcoxon rank-sum test will be used to compare the ranks of participants administered placebo versus sildenafil, overall and by sildenafil dose.

\section{Events of special interest}

The patients enrolled in this trial will be initially critically ill and deemed to require prolonged NICU care. Such individuals are known to have a high morbidity and mortality rate. The events (Table 4) will not be included in the primary safety outcome, but they are clinical ESI that may relate to safety and that will be tracked and reported in the trial results.

\section{Statistical analyses \\ General analysis considerations}

Analyses will be stratified by treatment assignment and dose groups: Cohort 1 Sildenafil, Cohort 2 Sildenafil, Cohort 3 Sildenafil, Overall Sildenafil and Overall Placebo. Patients dosed with IV or enteral formulations will be combined in analyses unless otherwise specified. Descriptive statistics will include number of observations, mean, standard deviation, median, minimum, and maximum for continuous variables or counts and percentages/proportions for categorical variables. Baseline assessment is the last non-missing value prior to first study drug administration.

\section{Sample size calculations}

The sample size of 30 in each dose group is sufficient to estimate hypotension incidence with sufficient precision. Table 5 provides widths for $95 \%$ Wilson confidence intervals in the dose groups of size 30 and the total sildenafil treatment cohort of 90 with different incidence rates. If hypotension occurs with an incidence rate of 0.05 , it has a $78 \%$ chance of being observed at least once in a dose group and a $99 \%$ chance of being observed at least once in the total sildenafil cohort.

\section{Safety analysis}

The primary safety endpoint is number and percent of hypotension events and will be summarized. Serious adverse events will be presented overall and by each Medical Dictionary for Regulatory Activities (MedDRA) system organ class and preferred term. Severity and relationship to sildenafil or placebo will be provided. ESI will be presented overall and summarized by participant characteristics. Prior and concomitant medications will be summarized by World Health Organization (WHO) drug class. Laboratory data, including change from baseline, will be tabulated by treatment groups.

\section{PK analysis}

A PK dataset will be created by the data coordinating center to include sildenafil and its primary metabolite desmethyl sildenafil (DMS) concentrations, dosing information, and clinical data for each subject enrolled in Cohorts 1,2 and 3 of the sildenafil trial. Sildenafil and DMS PK profiles will be constructed from the observed drug concentration-time data. The dataset will also include mean arterial pressure values.

Table 4 Events of Special Interest

\begin{tabular}{ll}
\hline Event & Definition \\
\hline Sepsis & Positive blood culture with an organism not typically considered a contaminant \\
Urinary tract infection & Positive urine culture with an organism not typically considered a contaminant \\
Bacterial meningitis & Positive cerebrospinal fluid culture with an organism not typically considered a contaminant \\
Retinopathy of Prematurity (ROP) & ROP requiring treatment with laser photocoagulation or an anti-VEGF drug \\
Seizures & Determined by the treating provider \\
Abnormal hearing test results & Based on testing during primary hospitalization \\
Systemic arterial or deep venous & Thrombosis requiring treatment with anticoagulation (e.g., heparin or low-molecular-weight heparin) \\
thrombosis & \\
Direct hyperbilirubinemia & Conjugated serum bilirubin $>$ 2.0 mg/dL \\
Elevated transaminases & ALT > 150 U/L or AST > 225 U/L \\
Endotracheal intubation & Intubation and transition from non-invasive to invasive ventilation, not due to planned surgical procedure (e.g., \\
\hline
\end{tabular}


Table 5 Widths for 95\% Wilson Confidence Intervals

\begin{tabular}{lllllll}
\hline $\boldsymbol{N}=\mathbf{3 0}$ & & & & $\boldsymbol{N}=\mathbf{9 0}$ & & \\
\cline { 1 - 1 } Rate & Width & $\mathbf{9 5 \%} \mathbf{C l}$ & & Rate & Width & $\mathbf{9 5 \% ~ C l}$ \\
\hline 0.1 & 0.22 & $0.04-0.26$ & & 0.1 & 0.13 & $0.05-0.18$ \\
0.2 & 0.28 & $0.10-0.37$ & & 0.2 & 0.16 & $0.13-0.29$ \\
0.3 & 0.31 & $0.17-0.48$ & & 0.3 & 0.19 & $0.22-0.40$ \\
\hline
\end{tabular}

Abbreviations: $\mathrm{Cl}$ confidence interval, $N$ number of participants

A population PK analysis will be performed using the sildenafil and DMS data to determine compartmental population PK parameters (i.e., clearance and volume) with the program NONMEM (Icon Solutions, Ellicott City, MD, USA). One and two compartment structural models will be explored for both sildenafil and DMS. Samples obtained after intravenous and enteral administration routes will be combined in a single model.

\section{Preliminary effectiveness analysis}

The presence or absence of $\mathrm{PH}$ will be determined using central review by blinded pediatric cardiologists of echocardiograms performed at the end of the study period. $\mathrm{PH}$ will also be graded as absent (normal), mild, moderate, or severe (Table 6). Results will be summarized by treatment assignment and dose group, and listed.

Risk and odds of $\mathrm{PH}$ in participants administered sildenafil versus placebo will be calculated (Risk Ratio, Odds Ratio [OR]), along with 95\% confidence intervals (CI), overall by treatment group and for each assigned sildenafil dose. Logistic regression models will be used to determine association between baseline $\mathrm{PH}$ risk and odds of developing $\mathrm{PH}$ for participants administered sildenafil versus placebo. Additional potential confounders may be included in the models if data allow, such as GA, PNA, sex, respiratory support, events of special interest, medications of special interest, and comorbidities. Repeated measures may be used to allow for inclusion of random effects for site and siblings if required by intraclass correlation coefficient.

\section{Discussion}

Despite the lack of safety and efficacy data in infants, neonatologists are increasingly using sildenafil to treat $\mathrm{PH}$ in premature infants. A review of data from the Pediatrix Medical Group Clinical Data Warehouse, a real-world data source representing approximately 20\% of US NICU admissions, found an exponential increase in sildenafil use by $>1000 \%$, second highest of all drugs from 2005 to 2010 [13]. Of the infants treated with sildenafil, $79 \%$ had BPD and $84 \%$ had $\mathrm{PH}$. Two case reports documented the successful resolution of $\mathrm{PH}$, measured by echocardiogram, in premature infants treated with sildenafil $[14,15]$. In a small case series, enteral sildenafil in 25 premature infants with lung disease resulted in improved hemodynamics by echocardiogram [16]. A retrospective case series of 23 preterm infants treated with enteral sildenafil found a substantial improvement in BPD-associated $\mathrm{PH}$ based on echocardiography in $71 \%$ of patients for whom echocardiographic data were complete $(15 / 21)$ [17]. These data confirm that neonatologists usually treat $\mathrm{PH}$ in infants with $\mathrm{BPD}$ and are likely unwilling to randomize to placebo; thus, a prevention approach is needed. This clinical trial will be essential to better understand the safety of this already commonly used medication in critically ill infants with severe BPD.

The strengths of this trial include a study design that will allow us to characterize the safety of sildenafil, confirm pharmacokinetics, and to explore the preliminary effectiveness and exposure-response relationship of

Table 6 Pulmonary hypertension composite score of echocardiographic measures

\begin{tabular}{|c|c|c|c|c|}
\hline \multirow{2}{*}{$\begin{array}{l}\text { Subjective } \\
\text { quantification of } \\
\text { RV pressures }\end{array}$} & \multirow{2}{*}{$\begin{array}{l}\text { Absolute criteria } \\
\text { Septal geometry in systole }\end{array}$} & \multicolumn{3}{|l|}{ Supportive criteria } \\
\hline & & Shunt direction $^{\mathrm{a}}$ & RV function ${ }^{\mathbf{b}}$ & RV/RA size ${ }^{c}$ \\
\hline $\begin{array}{l}\text { Normal } \\
(<1 / 3 \text { systemic })\end{array}$ & $\begin{array}{l}\text { Septum round } \\
\text { Eccentricity index }{ }^{d} \leq 1.0\end{array}$ & $L$ to $R$ & Normal & Normal \\
\hline $\begin{array}{l}\text { Mildly elevated } \\
(1 / 3-2 / 3 \text { systemic) }\end{array}$ & Septal geometry mildly distorted, Eccentricity index 1.01-1.20 & $L$ to $R$ & Normal & Mildly dilated \\
\hline $\begin{array}{l}\text { Moderately elevated } \\
(>2 / 3 \text { systemic) }\end{array}$ & Septum moderately distorted but not flat, Eccentricity index 1.21-1.4 & $L$ to $R$ & Mildly depressed & Mod. dilated \\
\hline $\begin{array}{l}\text { Severely elevated } \\
\text { ( } \geq \text { systemic) }\end{array}$ & Septum flat or bowing into left ventricle, Eccentricity index $>1.4$ & R to L & $\geq$ Mod. depressed & Severely dilated \\
\hline
\end{tabular}

${ }^{\mathrm{a} A c r o s s}$ a PFO, ASD or PDA

${ }^{\mathrm{b}}$ Measures of RV function to include: subjective estimates, RV fractional area change, tricuspid annular plane systolic excursion, RV strain and strain rate, Myocardial performance index

${ }^{\mathrm{c}} \mathrm{RV}$ to RA size based on subjective appearance

dLeft ventricular (LV) eccentricity index (LVEI) = LV antero-posterior diameter / LV septo-lateral diameter

Abbreviations: RV right ventricular, RA right atrial, PFO patent foramen ovale, $A S D$ atrial septal defect, $P D A$ patent ductus arteriosus, $L V$ left ventricular, $L V E I$ left ventricular eccentricity index

Reference: McCrary AW, Barker PCA, Torok RD, Spears TG, Li JS, Hornik CP, et al. agreement of an echocardiogram-based diagnosis of pulmonary hypertension in infants at risk for bronchopulmonary dysplasia among masked reviewers. J Perinatol. 2019;39 (2):248-55 
sildenafil in the treatment of BPD-associated PH using echocardiography. Additionally, the unique R61/R33 funding mechanism of the grant affords 2 years of funding for trial planning and site start-up, followed by 3 years of enrollment. This additional time allows the investigators to make the study better quality by conducting a community engagement studio, developing modeling for adjusted analyses of efficacy, and focusing on site engagement. A corresponding study to develop a prediction tool for $\mathrm{PH}$ in infants with BPD using machine learning is currently in process, and will compound the value that this trial provides. Furthermore, the global rank scores gathered in the tertiary outcome of the trial will provide valuable data for future studies and insight into the morbidity associated with BPD, PH, and prematurity.

The strategies utilized in this study are innovative to the field of infant clinical research, and create value for both patients and the community. We have utilized community engagement studios to optimize recruitment and retention, and will use electronic health records (EHR) and electronic consent procedures to increase efficiency in conducting a study of a rare disease in a vulnerable population. This clinical trial will make significant strides in understanding the use of sildenafil in premature infants with $\mathrm{PH}$, and provide a foundation for further exploration of utilization of sildenafil as a treatment modality for this condition.

Sildenafil is a promising potential intervention to prevent the development of $\mathrm{PH}$ in premature infants with BPD. Clinical trials of sildenafil specifically designed for premature infants are urgently needed. The current study will make significant contributions to scientific knowledge and safety of sildenafil in premature infants at risk for PH. Results from the study will be used by investigators to inform the design of a pivotal phase III efficacy trial.

\section{Supplementary Information}

The online version contains supplementary material available at https://doi. org/10.1186/s12887-020-02453-7.

Additional file 1: Appendix Table 1. Schedule of Events.

Additional file 2: Appendix Table 2. Global Rank Endpoint.

\footnotetext{
Abbreviations

ALT: Alanine Aminotransferase; AST: Aspartate Aminotransferase; BPD: Bronchopulmonary Dysplasia; Cl: Confidence Interval; CPAP: Continuous Positive Airway Pressure; DCRI: Duke Clinical Research Institute; DMS: Desmethyl Sildenafil; DSMB: Data Safety Monitoring Board; EHR: Electronic Health Records; ESI: Events of Special Interest; FDA: Food and Drug Administration; $\mathrm{FiO}_{2}$ : Fraction of Inspired Oxygen; GA: Gestational Age; GEE: Generalized Estimating Equations; IRB: Institutional Review Board; IV: Intravenous; LPM: Liters per Minute; MAP: Mean Arterial Pressure; MedDRA: Medical Dictionary for Regulatory Activities; NAVA: Neurally Adjusted Ventilatory Assist; NCPAP: Nasal Continuous Positive Airway Pressure; NHLBI: National Heart Lung and Blood Institute; NICHD: National
}

Institute of Child Health and Human Development; NICU: Neonatal Intensive Care Unit; NIPPV: Nasal Intermittent Positive Pressure Ventilation; OR: Odds Ratio; PD: Pharmacodynamics; PDA: Patent Ductus Arteriosus; PH: Pulmonary Hypertension; PK: Pharmacokinetics; PMA: Postmenstrual Age (gestational age plus postnatal age); ROP: Retinopathy of Prematurity; SAE: Serious Adverse Event; U/L: Units per Liter; VEGF: Vascular Endothelial Growth Factor; WHO: World Health Organization

\section{Acknowledgements}

Not applicable.

\section{Authors' contributions}

SS, TS, CE, ML, CH, and WJ made substantial contributions to the conception and design of the study. SS and WJ wrote the manuscript, and TS, CE, MB, $\mathrm{ML}$, and $\mathrm{CH}$ made edits. All authors have read and approved the final manuscript.

\section{Funding}

The trial proposed in this publication is supported by the National Heart, Lung, and Blood Institute (NHLBl; https://www.nhlbi.nih.gov/) of the National Institutes of Health (United States of America) under Award Number R61HL147833. The content is solely the responsibility of the authors and does not necessarily represent the official views of the National Center For Advancing Translational Sciences or the National Institutes of Health.

\section{Availability of data and materials}

Following completion of the study, the investigator may publish the results of this research in a scientific journal. All public presentations (abstracts, manuscripts, slides and text of oral or other presentations, and text of any transmission through any electronic media) by participating investigators, participating institutions, and DCC, will be reviewed by the Publication Committee per the Publication Committee charter.

All investigators funded by the $\mathrm{NIH}$ must submit or have submitted for them to the National Library of Medicine's PubMed Central an electronic version of their final, peer -reviewed manuscripts upon acceptance for publication, to be made publicly available no later than 12 months after the official date of publication. The study is a clinical trial and will comply with the NIH policy that establishes the expectation that all investigators conducting clinical trials funded in whole or in part by the $\mathrm{NIH}$ will ensure that these trials are registered at ClinicalTrials.gov, and that results of these trials are submitted to ClinicalTrials.gov.

\section{Ethics approval and consent to participate}

This protocol (Version Number 2.0; September 4, 2020) was approved by the Vanderbilt Human Research Protections Program (Vanderbilt University Medical Center) Single Institutional Review Board, IRB\# 201152, on 10/2/2020. Protocol modifications will be reviewed and approved by the IRB and updated at ClinicalTrials.gov. Written informed consent will be obtained by study personnel for all study participants by the participant's parent or guardian according to the IRB approved protocol.

\section{Consent for publication}

Not applicable.

\section{Competing interests}

The authors declare that they have no competing interests.

\section{Author details}

${ }^{1}$ Department of Pediatrics, School of Medicine, The University of North Carolina at Chapel Hill, Chapel Hill, NC, USA. ²Duke Clinical Research Institute, Durham, NC, USA. 'Department of Pediatrics, Duke University School of Medicine, Durham, NC, USA.

Received: 17 November 2020 Accepted: 1 December 2020

Published online: 14 December 2020

\section{References}

1. Stoll BJ, Hansen NI, Bell EF, Walsh MC, Carlo WA, Shankaran S, et al. Trends in care practices, morbidity, and mortality of extremely preterm neonates, 1993-2012. JAMA. 2015;314(10):1039-51. 
2. Higgins RD, Jobe AH, Koso-Thomas M, Bancalari E, Viscardi RM, Hartert TV, et al. Bronchopulmonary dysplasia: executive summary of a workshop. J Pediatr. 2018;197:300-8.

3. Jackson W, Hornik CP, Messina JA, Guglielmo K, Watwe A, Delancy G, et al. In-hospital outcomes of premature infants with severe bronchopulmonary dysplasia. J Perinatol. 2017;37(7):853-6.

4. Khemani E, McElhinney DB, Rhein L, Andrade O, Lacro RV, Thomas KC, et al. Pulmonary artery hypertension in formerly premature infants with bronchopulmonary dysplasia: clinical features and outcomes in the surfactant era. Pediatrics. 2007;120(6):1260-9.

5. Kim DH, Kim HS, Choi CW, Kim EK, Kim Bl, Choi JH. Risk factors for pulmonary artery hypertension in preterm infants with moderate or severe bronchopulmonary dysplasia. Neonatology. 2012;101(1):40-6.

6. Mourani PM, Sontag MK, Younoszai A, Miller JI, Kinsella JP, Baker CD, et al. Early pulmonary vascular disease in preterm infants at risk for bronchopulmonary dysplasia. Am J Respir Crit Care Med. 2015;191(1):87-95.

7. Collaco JM, Dadlani GH, Nies MK, Leshko J, Everett AD, McGrath-Morrow SA Risk factors and clinical outcomes in preterm infants with pulmonary hypertension. PLoS One. 2016;11(10):e0163904.

8. An HS, Bae EJ, Kim GB, Kwon BS, Beak JS, Kim EK, et al. Pulmonary hypertension in preterm infants with bronchopulmonary dysplasia. Korean Circ J. 2010:40(3):131-6.

9. Slaughter JL, Pakrashi T, Jones DE, South AP, Shah TA. Echocardiographic detection of pulmonary hypertension in extremely low birth weight infants with bronchopulmonary dysplasia requiring prolonged positive pressure ventilation. J Perinatol. 2011:31(10):635-40.

10. Bhat R, Salas AA, Foster C, Carlo WA, Ambalavanan N. Prospective analysis of pulmonary hypertension in extremely low birth weight infants. Pediatrics. 2012;129(3):e682-9.

11. Revatio (sildenafil) [package insert] New York, NY: Pfizer Labs; 2014. [Available from: https://www.accessdata.fda.gov/drugsatfda_docs/label/ 2014/021845s011,022473s004,0203109s002lbl.pdf.

12. McCrary AW, Barker PCA, Torok RD, Spears TG, Li JS, Hornik CP, et al. Agreement of an echocardiogram-based diagnosis of pulmonary hypertension in infants at risk for bronchopulmonary dysplasia among masked reviewers. J Perinatol. 2019;39(2):248-55.

13. Thompson EJ, Perez K, Hornik CP, Smith PB, Clark RH, Laughon M. Sildenafil exposure in the neonatal intensive care unit. Am J Perinatol. 2019;36(3):2627.

14. Caputo S, Furcolo G, Rabuano R, Basilicata AM, Pilla LM, De Simone A, et al. Severe pulmonary arterial hypertension in a very premature baby with bronchopulmonary dysplasia: normalization with long-term sildenafil. J Cardiovasc Med (Hagerstown). 2010;11(9):704-6.

15. Hon KL, Cheung KL, Siu KL, Leung TF, Yam MC, Fok TF, et al. Oral sildenafil for treatment of severe pulmonary hypertension in an infant. Biol Neonate. 2005;88(2):109-12.

16. Mourani PM, Sontag MK, Younoszai A, IVy DD, Abman SH. Clinical utility of echocardiography for the diagnosis and management of pulmonary vascular disease in young children with chronic lung disease. Pediatrics. 2008:121(2):317-25.

17. Trottier-Boucher MN, Lapointe A, Malo J, Fournier A, Raboisson MJ, Martin B, et al. Sildenafil for the treatment of pulmonary arterial hypertension in infants with Bronchopulmonary dysplasia. Pediatr Cardiol. 2015;36(6):125560

\section{Publisher's Note}

Springer Nature remains neutral with regard to jurisdictional claims in published maps and institutional affiliations.

Ready to submit your research? Choose BMC and benefit from:

- fast, convenient online submission

- thorough peer review by experienced researchers in your field

- rapid publication on acceptance

- support for research data, including large and complex data types

- gold Open Access which fosters wider collaboration and increased citations

- maximum visibility for your research: over $100 \mathrm{M}$ website views per year

At BMC, research is always in progress.

Learn more biomedcentral.com/submissions 